\title{
A constitutive model for multi-phase steels
}

\author{
E.S. Perdahcioğlu ${ }^{\dagger, *}$ and H.J.M. Geijselaers ${ }^{\dagger}$ \\ ${ }^{*}$ Materials innovation institute \\ ${ }^{\dagger}$ Faculty of Engineering Technology, Section of Applied Mechanics, \\ University of Twente, Postbus 217, 7500AE, Enschede, The Netherlands
}

\begin{abstract}
Mean-Field homogenization algorithms for materials involving two or more elasticplastic constituent phases are investigated. The Voigt, Reuss and Self consistent schemes which are directly applicable to multi-phase systems are implemented. The shortcomings of these schemes are accuracy for the former two and computational efficiency for the latter. A new interpolative model is proposed which is aimed to be both computationally efficient and accurate. The results of the models are studied on the material point level for a prescribed uniaxial tensile deformation. It is observed that the response computed by the proposed scheme closely matches that computed by the Self Consistent approach.
\end{abstract}

Keywords: Mean Field, homogenization, dual phase, TRIP, constitutive model

PACS: $46.35 .+\mathrm{z}$

\section{INTRODUCTION}

In the recent years, advanced materials such as Dual-Phase and TRIP steels have gained significant attraction. Due to their complex microstructure involving multiple phases and phase transformations, these materials possess exceptional mechanical properties. Furthermore, with slight changes in the heat treatment process during production, the phase distributions can be modified which results in different mechanical behavior. In order to exploit the properties of these materials in industrial applications, there is a need for physically based material models that take into account the relation between the microstructure and the mechanical response.

One way of achieving this goal is to use homogenization methods in which the microstructural effects directly contribute to the macroscopic behavior. In homogenization, generally, the microscopic (lower scale) features of the materials are considered, analyses are carried out and an averaging procedure is performed in order to return the results to the macroscale (upper scale). For the lower scale analyses usually the concept of an RVE (representative volume element) is utilized.

In the Mean-Field method instead of the fields of the variables, only the mean values over each subdomain are considered. The complementary problem then can be solved using an analytical approach such as Eshelby's solution of the inclusion problem [1]. Eshelby obtained the analytical solution for a simplified case where a single inclusion is assumed to be embedded in an infinitely large matrix. Furthermore, the solution is only for elastic materials.

In order to apply this solution in case of a finite-sized RVE with elastic-plastic material properties, different homogenization schemes have been proposed. The MoriTanaka scheme [2] relies on the Eshelby's solution to find the strain concentration in the 
inclusion. Another model that has been used for homogenization is the Self-Consistent scheme, first proposed by Kroner [3] and then used by Hill [4]. Hill also proposed to use the instantaneous elastic-plastic moduli of the phases as reference linearized comparison moduli that can be used with Eshelby's solution.

In modeling TRIP steels where more than two phases are involved, the Mori-Tanaka scheme cannot be applied directly. On the other hand, in [5], with the help of some simplifications, the applicability of this scheme has been investigated. One simplification is that the two of the phases, namely ferrite and bainite have been assumed to have the same mechanical properties which reduces the number of phases to three. The three phase homogenization problem is then solved using two approaches. The first one is to treat austenite as an inclusion within the ferrite-bainite matrix and then martensite within the ferrite-bainite-austenite matrix. The second approach is to treat both austenite and martensite as separate inclusions within half of the ferrite-bainite matrix and then solve the strain concentration for both halves. The results are demonstrated to agree well with experiments.

Here we present a different approach in which each phase can be treated as an inclusion in the overall composite material. This makes the model more generic and thus applicable to more variety of materials.

\section{MEAN FIELD HOMOGENIZATION}

Homogenization techniques are generally based on the concept of a representative volume element (RVE)[6]. An RVE represents a macroscopic point in the material (i.e. an integration point in a Finite Element model) as a finite sized inhomogeneous volume that is representative for the microstructure of the material. The aim therefore is to solve a complementary problem on the RVE and then use the results in macroscale.

The Mean-Field method is built on the concept of an imaginary RVE rather than an actual one. The inhomogeneities are treated as separate homogeneous domains in an aggregate of domains. The fields over the subdomains are represented by their averaged values. The complementary problem then is to solve for the interaction of the subdomains and find the new averages that satisfy the boundary conditions. This is usually carried out by using analytical solutions to the simplified versions of the problem [7].

The averaging operator which forms the foundation of the scale transition is defined as:

$$
\langle g(\mathbf{x})\rangle_{\omega}=\bar{g}=\frac{1}{V} \int_{\omega} g(\mathbf{x}) \mathrm{d} V=\sum_{k} f_{k}\langle g(\mathbf{x})\rangle_{\omega_{k}}, \quad f_{k}=\frac{V_{k}}{V} .
$$

where $g$ is any field defined over the domain, $\bar{g}$ is the macroscopic value and $\langle g(\mathbf{x})\rangle_{\omega}$ is the averaged value of $g$ over the coordinates $\mathbf{x}$ belonging to the volume $\omega$. It is clear the this formulation implies that the overall domain can be decomposed into smaller subdomains.

The averaging of strain and stress fields requires that the averaged mechanical work over the RVE is equal to the mechanical work that is calculated using the averaged stress and strain. This is known as the Hill-Mandel condition. According to [6], for this 
condition to be fulfilled, equilibrium and compatibility within the RVE must be satisfied. Furthermore, the boundary conditions applied on the surface of the RVE must be of the type that would produce uniform strain or stress within the RVE if the material were homogeneous. Supported by the Hill-Mandel condition, scale transition relations for stress and strain are given as:

$$
\overline{\boldsymbol{\sigma}}=\sum_{k} f_{k}\langle\boldsymbol{\sigma}\rangle_{\omega_{k}}, \quad \overline{\boldsymbol{\varepsilon}}=\sum_{k} f_{k}\langle\boldsymbol{\varepsilon}\rangle_{\omega_{k}} .
$$

The equations presented so far only provide the averaging operation once the fields are determined on the RVE. Using a Direct FE approach the fields can be computed by applying the macroscopic stress or strain in terms of surface boundary conditions (tractions and displacements, respectively) on the RVE $[8,9]$. After the fields are computed numerically, averaging is carried out and the results are transferred to the macroscale. In the Mean-Field homogenization schemes, instead of the fields, only the averages in different sub-domains are computed based on a number of assumptions which will be described in the following section.

Generally in the Mean-Field methods it is assumed that each constituent phase follows its own macroscopic material behavior [10,11]. With this assumption, the relation between the average stress and strain on the sub-domains on the RVE is supplied by continuum material models:

$$
\langle\dot{\boldsymbol{\sigma}}\rangle_{\omega_{k}}=\mathbb{C}_{k}:\langle\dot{\boldsymbol{\varepsilon}}\rangle_{\omega_{k}}
$$

where $\mathbb{C}$ is the elastic-plastic tangent modulus tensor.

The equations given so far are not sufficient to solve the homogenization problem. The remaining equations must supply the relation between the strains or stresses among the different phases. These equations can be written in terms of strain or stress concentration relations:

$$
\langle\boldsymbol{\varepsilon}\rangle_{\omega_{k}}=\mathbb{A}_{k}:\langle\boldsymbol{\varepsilon}\rangle_{\omega}, \quad\langle\boldsymbol{\sigma}\rangle_{\omega_{k}}=\mathbb{B}_{k}:\langle\boldsymbol{\sigma}\rangle_{\omega}
$$

where $\mathbb{A}$ and $\mathbb{B}$ are the fourth-order strain and the stress concentration tensors, respectively. In the case when all the concentration tensors of one type are known, the tensors for the other type can be determined. Mean-Field homogenization schemes differ from each other by the selection of these tensors. In the following sections the schemes that are studied are discussed.

\section{HOMOGENIZATION SCHEMES}

In this section the homogenization schemes that can be directly applied to multi-phase systems are presented. The most basic schemes are the bounds, Voigt (isostrain) and Reuss (isostress), which in the general case are not reliable in terms of accuracy. The Self Consistent scheme also applies to more than two phases as it was originally developed for polycrystals. However, because of its implicit nature it is computationally inefficient.

The isostrain condition, i.e. the Voigt scheme, yields an upper bound for the stiffness of the system. The strain concentration tensor for this scheme is unity and the stiffness 
of the composite is computed as the weighted sum of that of all the phases:

$$
\mathbb{A}=\mathbb{I}, \quad \mathbb{C}=\sum_{i} f_{i} \mathbb{C}_{i}
$$

In case of the isostress condition, i.e. the Reuss scheme, the stiffness of the system is on its lower bound and the stress concentration tensor is unity:

$$
\mathbb{A}_{i}=\mathbb{C}^{-1}: \mathbb{C}_{i}, \quad \mathbb{C}=\left(\sum_{i} f_{i} \mathbb{C}_{i}^{-1}\right)^{-1}
$$

For the bounds, the following consistency condition is always satisfied:

$$
\sum_{i} f_{i} \mathbb{A}_{i}=\mathbb{I} .
$$

In a multiphase system when the concentration tensors are calculated separately, then Equation (7) must be satisfied otherwise the partitioning of strain and the conservation of energy during scale transition are violated.

In the Self Consistent scheme, each phase is treated as an inclusion in the overall composite. The strain concentration for each phase is then calculated using Eshelby's theory accordingly:

$$
\mathbb{A}_{i}=\left[\mathbb{I}+\mathbb{E}:\left(\mathbb{C}^{-1}: \mathbb{C}_{i}-\mathbb{I}\right)\right]^{-1}, \quad \mathbb{C}=\sum_{i} f_{i} \mathbb{C}_{i}: \mathbb{A}_{i}
$$

where $\mathbb{E}$ is the Eshelby tensor and it is a function of the tangent modulus of the matrix phase and the shape of the inclusions. For descriptions of the Eshelby tensor for different geometries see for instance [12].

In this scheme the consistency condition Equation (7) is satisfied. On the other hand, it is clear that this scheme is inherently implicit and requires an iterative solution algorithm.

Here we introduce a new explicit scheme that is applicable for systems with more than two phases which has been introduced by the authors previously for two phase composites in [13]. The idea behind the model is to use an interpolation between the upper and lower bounds in order to determine the partitioning of strain into phases. The interpolation is defined as:

$$
\mathbb{H}_{i}=\left[\phi \mathbb{I}+(1-\phi)\left(\mathbb{C}^{r e}\right)^{-1}: \mathbb{C}_{i}\right]^{-1}, \quad \mathbb{C}^{r e}=\left(\sum_{i} f_{i} \mathbb{C}_{i}^{-1}\right)^{-1}, \quad \phi=\left(c_{1}+c_{2} f\right)^{c_{3}}
$$

where $\phi$ is the interpolation function. In order not to violate the theoretical bounds the following constraints must be imposed: $0 \leq c_{1}^{c_{3}} \leq 1,0 \leq\left(c_{1}+c_{2}\right)^{c_{3}} \leq 1$. Equation (9) does not satisfy the consistency condition given in Equation (7). This is because each phase has its concentrated based on its own stiffness and the other phases are not taken into account directly. On the other hand we propose that since the directions and the proportions of the strain are computed, each strain concentration tensor can be normalized to yield a consistent partitioning of the strain:

$$
\sum_{i} f_{i} \mathbb{H}_{i}=\mathbb{Q}, \quad \mathbb{A}_{i}=\mathbb{H}_{i}: \mathbb{Q}^{-1}
$$




\section{RESULTS}

The results of the schemes will be shown on a material point simulation. The problem is a uniaxial tensile deformation. All the phases are elastic-plastic with isotropic power law hardening behavior as $\sigma^{f}=\sigma^{0}+K\left(\varepsilon^{0}+\varepsilon^{p}\right)^{n}$. For the Self Consistent model, each phase is assumed spherical in shape. Additionally, for the calculation of the Eshelby tensor, the isotropic projection of the tangent modulus is used (for details see [11]). These selections make the Eshelby tensor isotropic.

Each phase is assumed to have identical isotropic elastic properties as; $E=210(\mathrm{GPa})$ and $v=0.3$. The plasticity material properties and the phase fractions of each phase are summarized in Table 1.

TABLE 1. Plasticity material parameters and phase fractions.

\begin{tabular}{rccccc}
\hline Phase & fraction & $\sigma^{y}(\mathrm{MPa})$ & $\varepsilon^{0}$ & $K(\mathrm{MPa})$ & $m$ \\
\hline Ferrite $(\alpha)$ & 0.50 & 291 & 0.008 & 742 & 0.1928 \\
Bainite & 0.10 & 800 & 0.001 & 2000 & 0.3 \\
Austenite $(\gamma)$ & 0.15 & 400 & 0.01 & 1500 & 0.31 \\
Martensite $\left(\alpha^{\prime}\right)$ & 0.25 & 1294 & 0.00016 & 2486 & 0.0747 \\
\hline
\end{tabular}

For the Bound Interpolation scheme the parameters of the interpolation function are selected as: $c_{1}=0.7, c_{2}=0.3, c_{3}=1.0$.

In Figure 1, the overall response of the four phase composite is shown. As expected the Voigt and Reuss schemes give the most stiff and the most compliant response, respectively. The Self Consistent scheme has no adjustable parameters and the response is solely dependent on the incremental stiffness of the phases. The Bound Interpolation scheme parameters have been selected to yield a response close to the Self Consistent scheme. With a different set of parameters the response can be modified.

It is clearly seen the proposed scheme can capture the stress and strain distribution to the phases accurately. The stress development in the phases in the orthogonal direction is observed to agree with the Self Consistent results. This is interesting to see since in the Bound Interpolation scheme the Eshelby tensor is not used. On the other hand, the assumption of spherical inclusions and isotropic matrix properties makes this tensor is isotropic. In the Bound Interpolation scheme, instead of the Eshelby tensor, a fourth order spherical tensor is used which is also isotropic but has only one value for both the deviatoric and the spherical directions (for more details see [13]).

\section{SUMMARY AND CONCLUSIONS}

The Mean Field homogenization scheme is investigated for applicability in modeling the mechanical response of multi-phase materials. The Self Consistent scheme can directly be used without a limitation on the number of phases as opposed to the Mori-Tanaka model which can only be used for two phase composites. A new scheme which was previously developed for two phase composites is generalized for multi-phase materials. The scheme is an interpolation of the upper and lower bound models with a scalar function. The function involves few number of parameters which determine the stiffness of the overall composite. It is shown that the partitioning of the stress and strain into different phases can be captured accurately once the Self Consistent model is considered as a reference. On the other hand with the proposed scheme a more detailed analysis such as ellipsoidal inclusions or anisotropic matrix properties cannot be captured. 


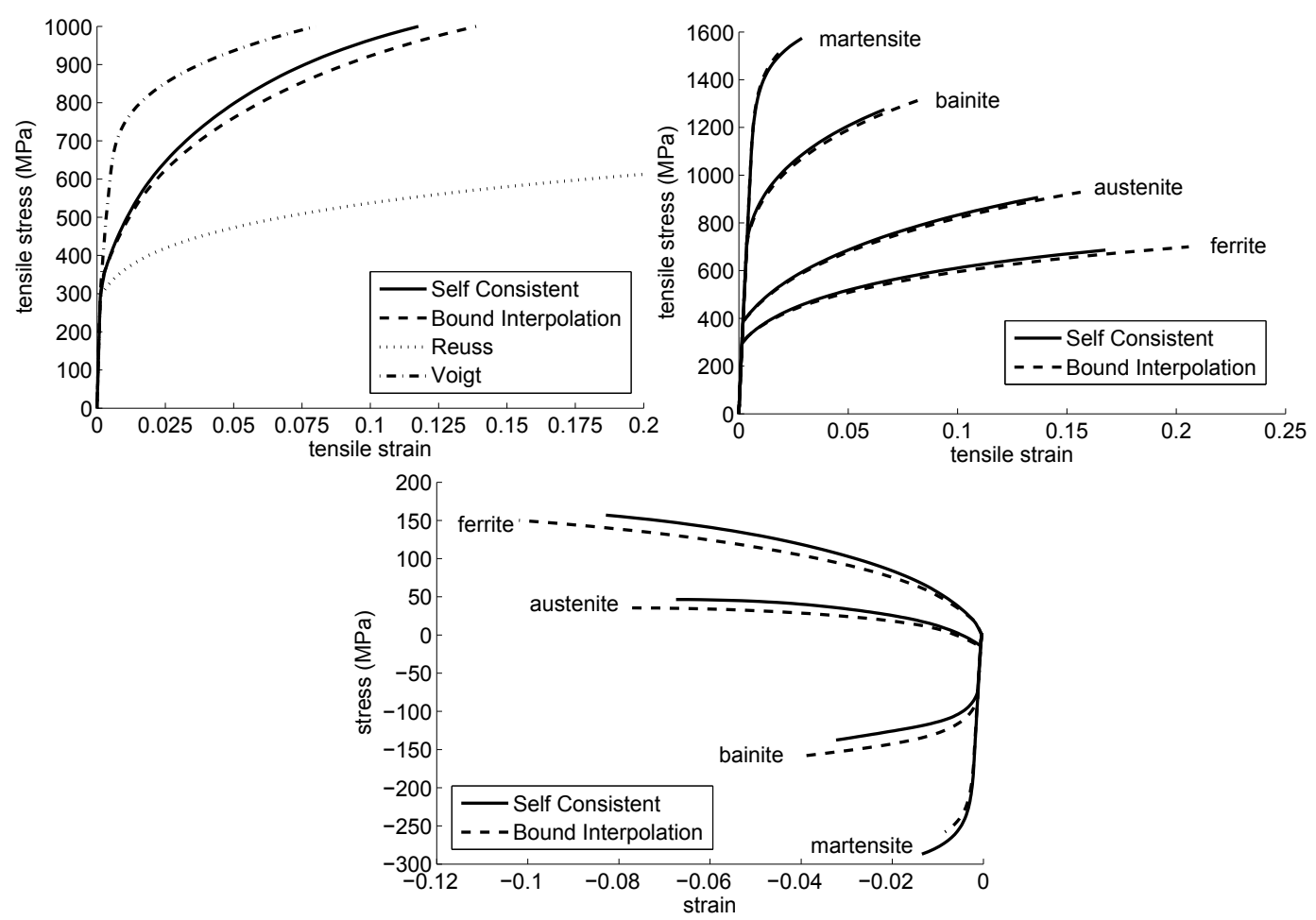

FIGURE 1. Comparison of the stress-strain relations obtained with different homogenization schemes under uniaxial tension. The overall response of the composite (top-left), the distribution of the stress and strain into the phases (top-right) and the stress and strain that develop in the orthogonal direction in each phase (bottom) are shown.

\section{ACKNOWLEDGMENTS}

This research was carried out under project number M63.1.09373 in the framework of the Research Program of the Materials innovation institute M2i (www.m2i.nl).

\section{REFERENCES}

1. J. Eshelby, Proceedings of the Royal Society of London. Series A 241, 376-396 (1957).

2. K. Tanaka, and T. Mori, Acta Metallurgica 18, 931-941 (1970).

3. E. Kröner, Zeitschrift für Physik 151, 504-518 (1958).

4. R. Hill, Journal of mechanics and physics of solids 13, 213-222 (1965).

5. L. Delannay, P. Jacques, and T. Pardoen, International Journal of Solids and Structures 45, 18251843 (2008).

6. R. Hill, Journal of mechanics and physics of solids 11, 357-372 (1963).

7. I. Doghri, Mechanics of Deformable Solids, Springer, 2000.

8. V. Kouznetsova, W. Brekelmans, and F. Baaijens, Computational Mechanics 27, 37-48 (2001).

9. V. Kouznetsova, M. Geers, and W. Brekelmans, Computer Methods in Applied Mechanics and Engineering 193, 5525-5550 (2004).

10. I. Doghri, and C. Friebel, Mechanics of Materials 37, 45-68 (2005).

11. I. Doghri, and A. Ouaar, International Journal of Solids and Structures 40, 1681-1712 (2003).

12. T. Mura, Micromechanics of defects in solids, Martinus Nijhoff Publishers, 1982.

13. E. Perdahcıoğlu, and H. Geijselaers, International Journal of Material Forming (2010), submitted. 